\title{
INTRABEAM SCATTERING AND WAKE FIELD EFFECTS IN LOW EMITTANCE ELECTRON RINGS
}

\author{
M. Venturini* \\ Stanford Linear Accelerator Center, Stanford University, Stanford, CA 94309, USA
}

\begin{abstract}
We present a model to study in a self-consistent way the interplay between intrabeam scattering and wake-field forces in low-emittance high-intensity electron storage rings. The regime of interest is that of the damping rings for the next generation of linear colliders.
\end{abstract}

\section{INTRODUCTION}

This work is being motivated by recent measurements at the ATF [1] - a prototype damping ring for future linear colliders - that have shown the relevance of the interplay between (multiple collision) intrabeam scattering (IBS) and longitudinal wake fields. The interplay stems from the fact that both effects help determine and in turn are affected by the bunch phase-space distribution. The proper framework to study the combination of these two effects is a VlasovFokker-Planck (VFP) equation. In plasma physics a FP modeling of particle collisions has long been established. Here we recall how to adapt the general FP description to the case of charged-particle beams and we then derive a reduced 1D VFP equation for the sole longitudinal motion with the inclusion of wake field forces and radiation effects. In this form the problem can be studied by numerically solving the reduced PDE for the longitudinal beam distribution and two ordinary differential equations for the evolution of the transverse emittances. A code for finding the solutions of the reduced problem has been written and an example of equilibrium distribution for a choice of parameters relevant for the ATF at moderate current is shown for illustration. Future work will include studying equilibria at high current and the effects of IBS on the onset of microwave instability and beam dynamics above the instability threshold.

\section{THE VFP EQUATION}

Our model of beam dynamics is the equation

$$
\frac{\partial f}{\partial s}+\{f, H\}=\left(\frac{\partial f}{\partial s}\right)_{c}+\mathrm{FP}_{r a d}(f)
$$

obeyed by the beam distribution function in the 6D phase space $f=f(\boldsymbol{X} ; s)$, with $\boldsymbol{X}=\left(x, p_{x}, y, p_{y}, z, p_{z}\right)$. The first two pairs of canonical coordinates, are relative to the motion in the horizontal and vertical planes, while $z$ describes the longitudinal displacement with respect to the synchronous particle and $p_{z}=\Delta p / p$ is the relative deviation of the total momentum from the design value; $s$ is the independent 'time-like' variable giving the location of a particle along the lattice. The Hamiltonian $H$ may include wake-field and possibly space charge forces in addition to the external forces provided by the magnetic lattice and RF cavities; $\{\cdot, \cdot\}$ are the Poisson brackets. The first term on the RHS represents the effect of collisions and the second that of synchrotron radiation. For the collision term we use a Fokker-Planck approximation (which can be obtained from the Boltzmann collision integral by doing a small angle expansion and retaining the lowest order terms [2] $),(\partial f / \partial \tilde{t})_{c} \simeq \tilde{F P}_{c}(\boldsymbol{X})$ with $(i, j=x, y, z)$

$$
\tilde{\mathrm{FP}}_{c}(\boldsymbol{X})=-\sum_{i} \frac{\partial}{\partial \tilde{p}_{i}}\left(\tilde{f} \tilde{D}_{i}\right)+\frac{1}{2} \sum_{i, j} \frac{\partial^{2}}{\partial \tilde{p}_{i} \partial \tilde{p}_{j}}\left(\tilde{f} \tilde{D}_{i j}\right)
$$

The tilde $\sim$ denotes quantities in the beam rest frame; in this frame $(\tilde{\boldsymbol{x}}, \tilde{\boldsymbol{p}}) \equiv \tilde{\boldsymbol{X}}$ are the actual position and mechanical momentum. Drift $\tilde{D}_{i}$ and diffusion $\tilde{D}_{i j}$ coefficients can be written as

$$
\begin{aligned}
\tilde{D}_{i}(\tilde{\boldsymbol{x}}, \tilde{\boldsymbol{p}}) & =\Gamma \frac{\partial}{\partial \tilde{p}_{i}} \int \frac{\tilde{f}\left(\tilde{\boldsymbol{x}}, \tilde{\boldsymbol{p}}^{\prime}\right)}{\left|\tilde{\boldsymbol{p}}-\tilde{\boldsymbol{p}}^{\prime}\right|} d^{3} \tilde{\boldsymbol{p}}^{\prime} \\
\tilde{D}_{i j}(\tilde{\boldsymbol{x}}, \tilde{\boldsymbol{p}}) & =\frac{\Gamma}{2} \frac{\partial^{2}}{\partial \tilde{p}_{i} \partial \tilde{p}_{j}} \int \tilde{f}\left(\tilde{\boldsymbol{x}}, \tilde{\boldsymbol{p}}^{\prime}\right)\left|\tilde{\boldsymbol{p}}-\tilde{\boldsymbol{p}}^{\prime}\right| d^{3} \tilde{\boldsymbol{p}}^{\prime}
\end{aligned}
$$

where $\Gamma=8 \pi m^{3} c^{4} r_{c}^{2} \log \Lambda_{c}$, with $r_{c}$ being the classical radius of the particle and $\log \Lambda_{c} \simeq \log \left(2 / \theta_{m}\right)$ is the so called Coulomb logarithm. For emittance dominated beams the minimum scattering angle $\theta_{m}$ is determined by bunch sizes. The integrals in (3) and (4) are known in plasma physics as 'Rosenbluth potentials' although it appears they were first derived by Landau.

To obtain the Fokker-Planck equation in the lab frame it is just a matter of applying the proper transformation to Eq. (2). In the paraxial approximation the transformation from the lab frame coordinates $\boldsymbol{X}$ to the beam frame coordinates $\tilde{\boldsymbol{X}}$ is just a scaling, which can be represented by a diagonal matrix $\mathrm{M}, \tilde{\boldsymbol{X}}=\mathrm{M} \boldsymbol{X}$. Invariance of the number of scattered particles as recorded in the two frames $\mathrm{FP}_{c}(\boldsymbol{X}) d^{6} \boldsymbol{X} d s=\tilde{\mathrm{FP}}_{c}(\tilde{\boldsymbol{X}}) d^{6} \tilde{\boldsymbol{X}} d \tilde{t}$ permits writing the Fokker-Planck term in the lab frame in terms of (2): $\mathrm{FP}_{c}(\boldsymbol{X})=\tilde{\mathrm{FP}_{c}}(\mathrm{M} \boldsymbol{X}) /|\operatorname{det} \mathrm{M}| \gamma_{0} v_{0}$, having used $d s=v_{0} d t$, where $v_{0}$ is the design beam velocity in the lab frame, and the relativistic time dilation $d t=\gamma d \tilde{t}$. If we denote as $\mathrm{N}$ the part of transformation $\mathrm{M}$ relative to the momenta only we have $\mathrm{N}_{11}=\mathrm{N}_{22}=p_{0}$ and $\mathrm{N}_{33}=p_{0} / \gamma_{0}$ (off-diagonal terms vanish) and the VFP equation in the Lab frame can be written as

$$
\frac{\partial f}{\partial s}+\{f, H\}=-\sum_{i} \frac{\partial}{\partial p_{i}}\left(f D_{i}\right)+\frac{1}{2} \sum_{i, j} \frac{\partial^{2}}{\partial p_{i} \partial p_{j}}\left(f D_{i j}\right),
$$

\footnotetext{
*venturin@slac.stanford.edu
} 
with

$$
\begin{aligned}
D_{i} & =\frac{\Gamma}{\gamma_{0}^{2} v_{0} \mathrm{~N}_{i i}^{2}} \frac{\partial}{\partial p_{i}} \int \frac{f\left(\boldsymbol{x}, \boldsymbol{p}^{\prime}\right)}{\left|\mathrm{N}\left(\boldsymbol{p}-\boldsymbol{p}^{\prime}\right)\right|} d^{3} \boldsymbol{p}^{\prime}, \\
D_{i j} & =\frac{\Gamma}{2 \gamma_{0}^{2} v_{0} \mathrm{~N}_{i i}^{2} \mathrm{~N}_{j j}^{2}} \frac{\partial^{2}}{\partial p_{i} \partial p_{j}} \int f\left(\boldsymbol{x}, \boldsymbol{p}^{\prime}\right)\left|\mathrm{N}\left(\boldsymbol{p}-\boldsymbol{p}^{\prime}\right)\right| d^{3} \boldsymbol{p}^{\prime},
\end{aligned}
$$

having also made use of $f(\boldsymbol{X})=|\operatorname{det} M| \tilde{f}(\mathrm{M} \boldsymbol{X})=$ $p_{0}^{3} \tilde{f}(\mathrm{M} \boldsymbol{X})$. For a discussion on the range of applicability of the VFP equation we refer the reader to e.g. [2]. The familiar IBS growth rates for the emittances [3] can obtained after multiplying both sides of Eq. (5) by second powers of momenta and integrating over the phase space variables under the assumption that the distribution function $f$ is gaussian.

\section{A REDUCED 1-D VFP EQUATION}

For the beam we assume a distribution of the form

$$
f(\boldsymbol{X})=\frac{N g\left(z, p_{z}\right)}{(2 \pi)^{2} \varepsilon_{x} \varepsilon_{y}} \exp \left[-S^{x}(\boldsymbol{X})-S^{y}(\boldsymbol{X})\right],
$$

with the horizontal and linear invariants given by

$$
\begin{aligned}
S^{x} & =\left[\beta_{x}\left(p_{x}-p_{z} \eta_{x}^{\prime}\right)^{2}+2 \alpha_{x}\left(x-p_{z} \eta_{x}\right)\left(p_{x}-p_{z} \eta_{x}^{\prime}\right)\right. \\
& \left.+\gamma_{x}\left(x-p_{z} \eta_{x}\right)^{2}\right] / 2 \varepsilon_{x} .
\end{aligned}
$$

(Similar expression for $S_{y}$ ). Here $\alpha_{x}, \beta_{x}, \gamma_{x}$ are the Courant functions and $\eta_{x}$ is the dispersion function. Let the Hamiltonian $H_{z}$ for the longitudinal motion be that of an ultra-relativistic electron bunch experiencing linear RF forces and single-turn wake fields. Such a Hamiltonian reads [4]

$$
\begin{aligned}
H_{z}= & \frac{1}{2} p_{z}^{2} \alpha_{c}+\frac{1}{2 \alpha_{c}}\left(\frac{\nu_{s}}{R}\right)^{2} z^{2}+ \\
& I \int_{z}^{\infty} d z^{\prime \prime} \int_{-\infty}^{\infty} d z^{\prime} W\left(z^{\prime \prime}-z^{\prime}\right) \rho_{z}(z),
\end{aligned}
$$

where $W\left(z^{\prime \prime}-z^{\prime}\right) / 2 \pi R$ has the meaning of averaged (over one turn) longitudinal electric field per unit charge acting on a test particle in $z^{\prime \prime}$ due to a point source at $z^{\prime} ; R$ is the machine radius; $\rho_{z}=\int d p_{z} g\left(z, p_{z}\right)$ is the longitudinal beam density; $\nu_{s}$ the synchrotron oscillation tune; $\alpha_{c}$ the momentum compaction and finally $I=e^{2} N / 2 \pi R c p_{0}$. The reduced VFP equation obeyed by $g$ is obtained from Eq. (5) by integrating with respect to the transverse coordinates. This leads to

$$
\frac{\partial g}{\partial s}+\left\{g, H_{z}\right\}=-\frac{\partial}{\partial p_{z}}\left(g \hat{D}_{z}\right)+\frac{1}{2} \frac{\partial^{2}}{\partial p_{z}^{2}}\left(g \hat{D}_{z z}\right)+\mathrm{FP}_{\mathrm{rad}}(g),
$$

where the drift and diffusion coefficients due to IBS are

$$
\begin{aligned}
\hat{D}_{z} & =\int d \boldsymbol{x}_{\perp} d \boldsymbol{p}_{\perp} f(\boldsymbol{X}) D_{z}(\boldsymbol{x}, \boldsymbol{p}), \\
\hat{D}_{z z} & =\int d \boldsymbol{x}_{\perp} d \boldsymbol{p}_{\perp} f(\boldsymbol{X}) D_{z z}(\boldsymbol{x}, \boldsymbol{p}),
\end{aligned}
$$

with $D_{z}$ and $D_{z z}$ given by the expressions at the end of the previous Section. The last term on the RHS of (9) represents the Fokker-Planck term associated with the effects of radiation on the longitudinal motion. It can be written as

$$
\mathrm{FP}_{\mathrm{rad}}(g)=\frac{2}{c \tau_{p}^{r a d}} \frac{\partial}{\partial p_{z}}\left(p_{z} g+\sigma_{p 0}^{2} \frac{\partial}{\partial p_{z}} g\right),
$$

where $\tau_{p}^{r a d}$ is the longitudinal damping time and $\sigma_{p 0}$ the natural (relative) momentum spread of a bunch at equilibrium due to sole radiation effects. The results of integration in (10) and (11) are best written in terms of the auxiliary function

$$
\mathcal{F}_{\alpha}(u)=\int_{0}^{\infty} d \lambda \frac{\lambda^{\alpha} e^{-\left[A_{33}+\lambda-A_{13} /\left(A_{11}+\lambda\right)\right] u^{2} / 4}}{\left(\lambda+\beta_{x} / \varepsilon_{x}\right)^{\frac{1}{2}}\left(\lambda+\beta_{y} / \varepsilon_{y}\right)^{\frac{1}{2}}} .
$$

After defining $A=N r_{c}^{2} \log \left(\Lambda_{c}\right) / 2 \sqrt{\pi} \beta_{0}^{4} \gamma_{0}^{5} \varepsilon_{x} \varepsilon_{y}$ and $u_{z}=$ $\left(p_{z}-p_{z}^{\prime}\right) / \gamma_{0}$ we have

$$
\begin{aligned}
\hat{D}_{z} & =-A \int_{-\infty}^{\infty} d p_{z}^{\prime} g\left(z, p_{z}^{\prime}\right) u_{z} \mathcal{F}_{\frac{1}{2}}\left(u_{z}\right), \\
\hat{D}_{z z} & =\frac{A}{2} \int_{-\infty}^{\infty} d p_{z}^{\prime} g\left(z, p_{z}^{\prime}\right)\left[2 \mathcal{F}_{-\frac{1}{2}}\left(u_{z}\right)-u_{z}^{2} \mathcal{F}_{\frac{1}{2}}\left(u_{z}\right)\right] .
\end{aligned}
$$

with $A_{11}=\beta_{x} \varepsilon_{x}, A_{122}=\beta_{y} \varepsilon_{y}, A_{33}=\mathcal{H}_{x} / \varepsilon_{x}$ and $A_{13}=$ $-\left(\beta_{x} \eta_{x}^{\prime}+\alpha_{x} \eta_{x}\right) \varepsilon_{x}$. The RHS of the above expressions are understood to be averaged over the lattice.

Eq. (9) is not self-contained because the IBS drift and diffusion coefficients depend on the transverse emittances. The evolution of the transverse emittances can be determined by solving a pair of ODE's using the familiar expressions for the IBS growth [3], radiation damping and excitation rates. At each time step these equations require specification of longitudinal rms bunch size and momentum spread, which are determined from the solution $g$ of (9). Notice that this scheme is not completely self-consistent because the familiar IBS growth rates are derived on the assumption that the beam distribution is gaussian while $g$, in general, is not.

\section{NUMERICAL STUDY}

To solve the VFP equation (9) with the FP term including both radiation and IBS effect we follow the method proposed in [4] with a modified implementation to improve efficiency. The longitudinal distribution function $g$ is represented on a $N \times N$ cartesian grid (for the calculations reported below $N=201)$. Evaluations of the Vlasov and the Fokker-Planck part are carried out separately (operator splitting). While the FP part is treated using the Crank-Nicholson method, propagation of the distribution function under the actions of the Vlasov part of Eq. (9) is done using the map $M \equiv M_{s \rightarrow s+\delta s}$ expressing the Hamiltonian flow from 'time' $s$ to $s+\delta s, g\left(z, p_{z} ; s+\delta s\right)=$ $g\left(M^{-1}\left(z, p_{z}\right) ; s\right)$. For $M$ we used a $2^{\text {nd }}$ order symplectic approximation, including the action of both the external and wake forces: this an improvement over the first order 
integrator in [4]. A third order polynomial interpolation is carried out at each time step to evaluate $g$ on the grid points [in general, if $\left(z, p_{z}\right)$ is a grid point $M^{-1}\left(z, p_{z}\right)$ is not]. The evolution of the emittances is calculated using a second order Runge-Kutta method.

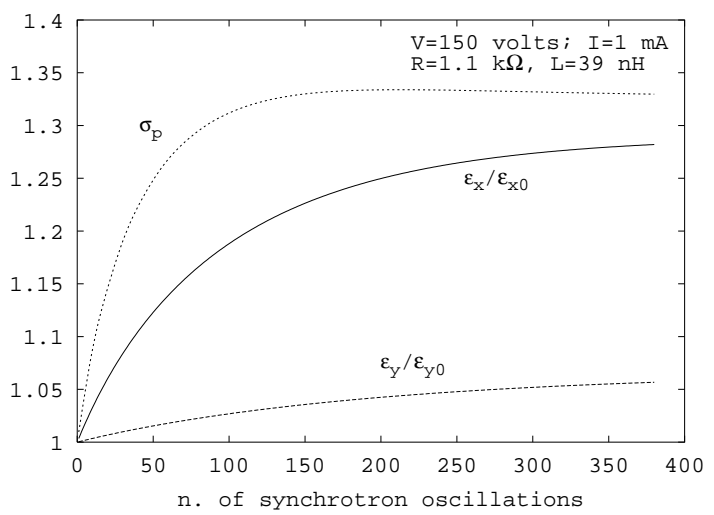

Figure 1: Evolution of relative momentum spread $\sigma_{p}=$ $\sqrt{\left\langle p_{z}\right\rangle^{2}} / \sigma_{p 0}$ and transverse emittances; $\mathrm{I}=1.0 \mathrm{~mA}$.

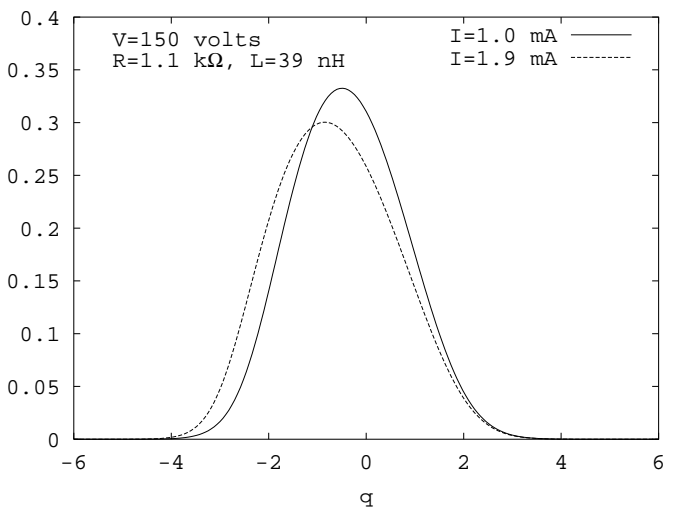

Figure 2: Equilibrium distributions in the absence of IBS (Haissinski solutions) for $\mathrm{I}=1.0$ and $1.9 \mathrm{~mA} ; q=z / \sigma_{s 0}$ is the scaled longitudinal distance, $\sigma_{s 0}$ the natural bunch length in the zero-current limit.

For the purpose of illustrating the method we show an example of solution representing a beam distribution approaching equilibrium. The various parameters are relative to the ATF $[1,5]$. The wake field potential $W(\tau)$ is approximated by a RL model in the form $W(\tau)=c \hat{R} \delta(\tau)+$ $c^{2} \hat{L} \delta^{\prime}(\tau)$ where $\hat{R}$ and $\hat{L}$ are the resistive and inductive part of the impedance and $\delta$ is the Dirac function. An estimate of $\hat{R}$ and $\hat{L}$ is reported in [5]. For this calculation $\hat{R}=1.1$ $\mathrm{k} \Omega$ and $\hat{L}=39 \mathrm{nH}$. The example presented in the picture is relative to operations at $\mathrm{V}=150$ volts corresponding to a synchrotron oscillation frequency of $\omega_{s}=44.4 \mathrm{kHz}$. The radiation longitudinal damping time is about 150 synchrotron oscillation periods. Fig. 1 shows the evolution of relative momentum spread and emittances for a circulating current of $I=1 \mathrm{~mA}$ corresponding to $N \simeq 2.9 \times 10^{9}$ par- ticles/bunch. The initial distribution $(s=0)$ is set to be the equilibrium distribution both longitudinally and transversally in the absence of IBS with the longitudinal profile of the distribution (Haissinski solution) shown in Fig. 2 (the distribution in $p_{z}$ is a gaussian). The initial values of the transverse emittances having assumed a $1 \% x / y$-coupling [1] are $\varepsilon_{x 0}=10^{-9} \mathrm{~m}$ and $\varepsilon_{y 0}=10^{-11}$. The growth in the bunch sizes in all three dimensions shown in the picture is entirely due to IBS. Afer about $n_{p}=150$ synchrotron oscillation periods (corresponding to the longitudinal radiation damping time) the longitudinal distribution appears to be already close to equilibrium (the transverse distribution is not settled yet because of the longer transverse damping times). The relative momentum spread distribution at $n_{p}=380$ is reported in Fig. 3 (solid line) together with an equivalent gaussian distribution having the same second moment (the initial $p$-distribution is also displayed). The picture shows that the momentum distribution (which is not far from equilibrium) is different from but still very close to being gaussian.

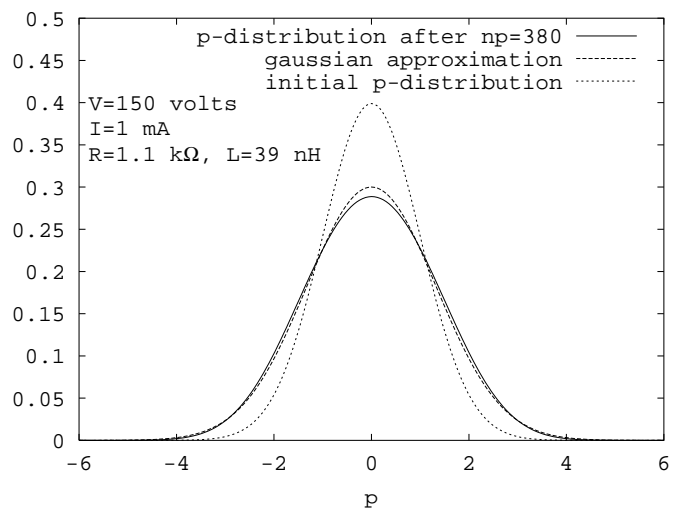

Figure 3: Relative momentum spread distribution ( $p=$ $p_{z} / \sigma_{p 0}$ ) after 380 synchrotron oscillation periods (solid line).

I would like to thank A. Kabel, R. Ruth, and in particular R. Warnock and K. Bane for useful discussions and assistance. Work supported by DOE contract DE-AC0376 SF0051.

\section{REFERENCES}

[1] K. Bane, et al., SLAC Rep. SLAC-AAS-97, Stanford (2000); K. Bane, et al., SLAC Rep. SLAC-AP-135, Stanford (2000).

[2] R. Liboff, Kinetic Theory, Wiley, (1998); J. Binney and S. Tremaine, Galactic Dynamics, Princeton University Press, Princeton (1987).

[3] J. Bjorken and S. Mtingwa, Part. Accel., 13, 115 (1983); M. Venturini, Study of Intrabeam Scattering in Low-Energy Electron Rings, these Proceedings.

[4] R. Warnock and J. Ellison, in The Physics of High Brightness Beams, J. Rosenzweig and L. Serafini Eds., World Scientific, Singapore (2000).

[5] K. Bane, et al., SLAC Rep. SLAC-PUB-8846, Stanford 2001. 